\title{
Simple and Scalable Streaming: The GRETA Data Pipeline
}

\author{
Mario Cromaz ${ }^{1, * *}$, Eli Dart $^{2, * * *}$, Eric Pouyoul ${ }^{2, * * * *}$, and Gustav R. Jansen ${ }^{3, \dagger}$ \\ ${ }^{1}$ Nuclear Science Division, Lawrence Berkeley National Laboratory, CA, USA \\ ${ }^{2}$ Energy Sciences Network (ESnet), Lawrence Berkeley National Laboratory, CA, USA \\ ${ }^{3}$ National Center for Computational Sciences, Oak Ridge National Laboratory, TN, USA
}

\begin{abstract}
The Gamma Ray Energy Tracking Array (GRETA) is a state of the art gamma-ray spectrometer being built at Lawrence Berkeley National Laboratory to be first sited at the Facility for Rare Isotope Beams (FRIB) at Michigan State University. A key design requirement for the spectrometer is to perform gamma-ray tracking in near real time. To meet this requirement we have used an inline, streaming approach to signal processing in the GRETA data acquisition system, using a GPU-equipped computing cluster. The data stream will reach 480 thousand events per second at an aggregate data rate of 4 gigabytes per second at full design capacity. We have been able to simplify the architecture of the streaming system greatly by interfacing the FPGA-based detector electronics with the computing cluster using standard network technology. A set of highperformance software components to implement queuing, flow control, event processing and event building have been developed, all in a streaming environ-
\end{abstract}

${ }^{*}$ This work was supported by the Director, Office of Science, Office of Advanced Scientific Computing Research (ASCR), of the U.S. Department of Energy under Contract No. DE-AC02-05CH11231.

This manuscript has been authored by an author at Lawrence Berkeley National Laboratory under Contract No. DE-AC02-05CH11231 with the U.S. Department of Energy. The U.S. Government retains, and the publisher, by accepting the article for publication, acknowledges, that the U.S. Government retains a non-exclusive, paid-up, irrevocable, world-wide license to publish or reproduce the published form of this manuscript, or allow others to do so, for U.S. Government purposes.

Notice: "This manuscript has been authored by UT-Battelle, LLC under Contract No. DE-AC05-00OR22725 with the U.S. Department of Energy. The United States Government retains and the publisher, by accepting the article for publication, acknowledges that the United States Government retains a non-exclusive, paid-up, irrevocable, worldwide license to publish or reproduce the published form of this manuscript, or allow others to do so, for United States Government purposes. The Department of Energy will provide public access to these results of federally sponsored research in accordance with the DOE Public Access Plan (http://energy.gov/downloads/doe-public-access-plan)."

Disclaimer: This document was prepared as an account of work sponsored by the United States Government. While this document is believed to contain correct information, neither the United States Government nor any agency thereof, nor the Regents of the University of California, nor any of their employees, makes any warranty, express or implied, or assumes any legal responsibility for the accuracy, completeness, or usefulness of any information, apparatus, product, or process disclosed, or represents that its use would not infringe privately owned rights. Reference herein to any specific commercial product, process, or service by its trade name, trademark, manufacturer, or otherwise, does not necessarily constitute or imply its endorsement, recommendation, or favoring by the United States Government or any agency thereof, or the Regents of the University of California. The views and opinions of authors expressed herein do not necessarily state or reflect those of the United States Government or any agency thereof or the Regents of the University of California.

**e-mail: mcromaz@lbl.gov

***e-mail: dart@es.net

****e-mail: lomax@es.net

†e-mail: jansengr@ornl.gov 
ment which matches detector performance. Prototypes of all high-performance components have been completed and meet design specifications.

\section{Introduction}

The Gamma-Ray Energy Tracking Array (GRETA)[1] is a large scale gamma-ray spectrometer that will be used to perform nuclear structure and nuclear astrophysics studies at facilities such as the Facility for Rare Isotope Beams (FRIB)[2] and at the Argonne Tandem Linear Accelerator System (ATLAS)[3]. This detector uses gamma-ray tracking[4, 5], a new technology where the interaction points of the incident scattering gamma-rays can be localized in the HPGe detector volume to mm precision so that Compton tracking can be performed. This allows for the device to have very high gamma-ray efficiency while maintaining a good peak-to-total ratio, thus extending the physics reach of the instrument.

A unique aspect of GRETA is the need to process the data online with a latency of less than ten seconds. The requirement is driven by the relatively short duration (1-5 days) of the experiments, often with multiple beam and target combinations that results in constant changes to the experimental setups and device configurations. To make optimal use of the available machine time, experimenters must be able to debug and optimize their experiments in real time, without resorting to offline analysis. Meeting this latency requirement is a challenging problem as the data analysis procedures to locate the interaction points of the gamma rays in the HPGe volume from the raw digitized waveforms to support tracking are both data intensive and computationally complex. To address this we have adopted a streaming data acquisition system which we believe generalizes to a large class of event-based experiments. Furthermore the data transport aspects of the system are abstracted from the specific analysis procedures. Our results on prototype implementations indicate that the design is both feasible and scalable.

Streaming architectures when applied to physics instruments can be complex. Previous work carried out on the GRETINA[6] detector, a currently-operating demonstrator for gamma-ray tracking technology, required a complex hardware and network infrastructure that included purpose-built VME-based digitizers, embedded readout computers, custom protocols for communication, and static provisioning of the cluster resources. Moving from GRETINA to GRETA, there was a clear need for higher throughput, but also a need for a simpler, more maintainable design that allows for dynamic provisioning of computing resources. Our approach to this problem has been to apply standards-based networking as early in the data acquisition process as possible (at the level of front-end FPGAs) and leverage widely used communication protocols and libraries.

In this paper we will discuss the GRETA network environment, and then the architecture of the GRETA data pipeline and its requirements. This will be followed by a detailed description of the prototypes of the primary data pipeline components: the forward buffer, the event processing component, and the event builder, as well as the common communication library which binds them together. Benchmark results carried out on the prototypes of these three components using realistic traffic simulations will be given to show the performance scope of the system. We will end with a brief discussion of future work and possible generalizations of the system to other use cases.

\section{The GRETA Network Environment}

All of the data pipeline components, as well as the control system components, of the GRETA environment are interconnected by a standards-based high-performance network. GRETA 
has an unusual requirement in that it is assumed the instrument can move between experimental facilities as is the case with its predecessor GRETINA. This requires that the network be self-contained, with minimal reconfiguration required when moving GRETA between host facilities. A further requirement is that GRETA can operate in the presence of external network connectivity interruptions. This requires that the GRETA network environment selfhost a number of standard services (e.g. DNS, NTP, local Docker registry, etc.). In short, GRETA must be self-sufficient for all services required for experiment operation.

Sites hosting GRETA will have two primary physical locations for computing hardware deployment: one is the experiment hall, where the detectors and electronics are deployed, and the other is a facility computing room, where the Forward Buffers, Event Builder, Event Processing Cluster, storage systems, cluster management, and other servers are deployed. Switches which receive high-speed UDP traffic from forward buffers are deeply buffered to reduce the probability of packet loss due to incast congestion. The controls network and data network are separate from each other (so that neither interferes with the other), but both networks are present in both locations. The two locations are connected with two redundant link pairs, one for the controls network and one for the data network. In addition, in the computing cluster area there is a management network for managing the Linux systems in that part of the network. The controls network uses the EPICS[7] control system. A full description of the GRETA control systems will be published in a future paper.

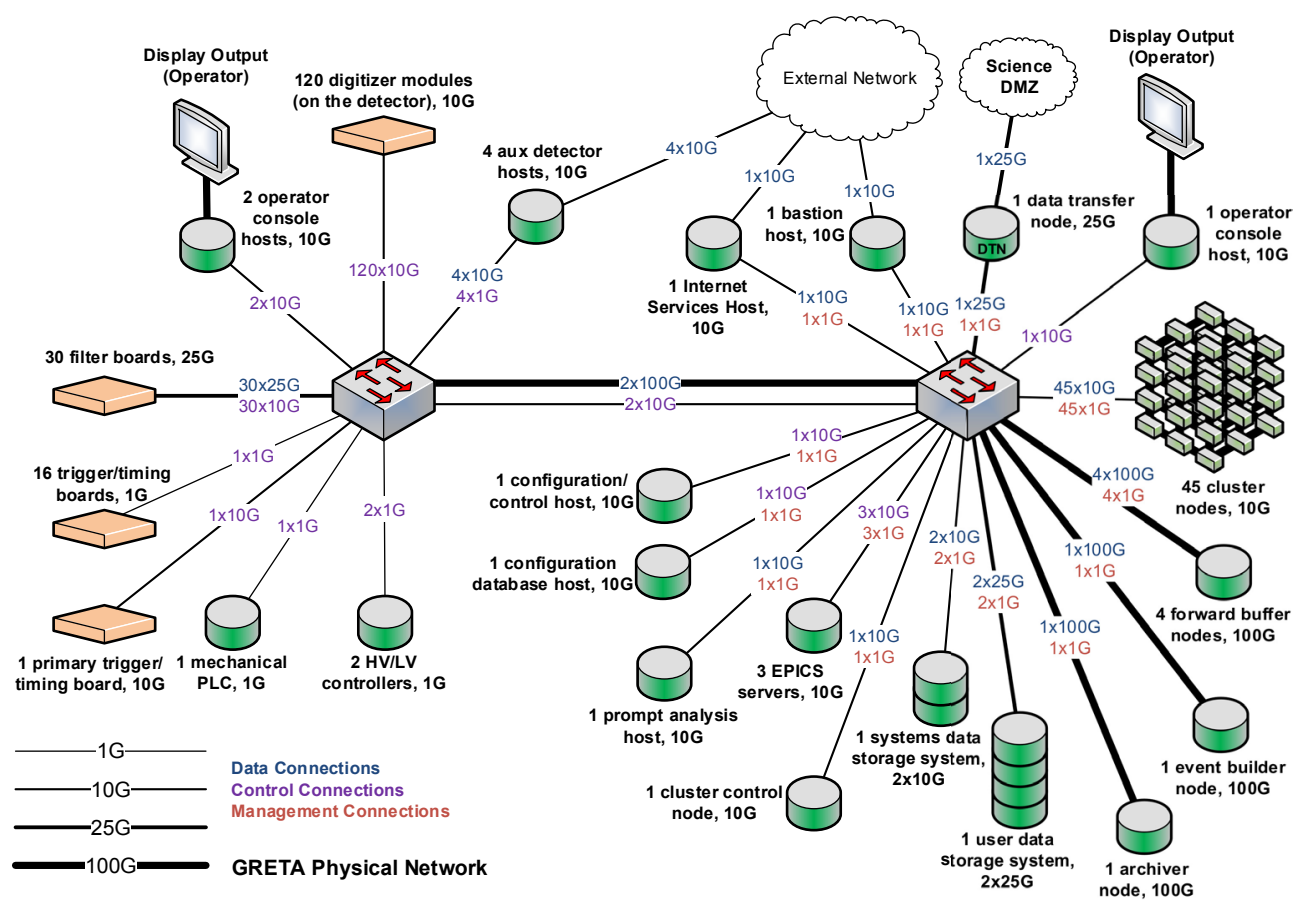

Figure 1. A physical map of the future GRETA network design. Connection counts, as well as control, data, and management networks are shown.

As is the case with any modern computing environment, cybersecurity is of great importance to GRETA. Because the timing of future security vulnerabilities cannot be determined, and the GRETA data pipeline must be able to operate during experiments, the security architecture assumes that security vulnerabilities are present in one or more systems within the 
GRETA environment, and that GRETA must be able to operate safely despite the vulnerabilities. The primary mechanism for protecting the GRETA environment is network isolation. This means that only a small set of specific systems are allowed to connect to the Internet at all, and those systems are not part of the data pipeline (for example, bastion hosts for remote login by system administrators are allowed, but these run a small set of specific services with a tight security policy, and are not involved in experiment operation or data processing). There is one exception to this, which is the computing system used to support an auxiliary detector. The approach taken here is to limit privilege and restrict services available to the auxiliary detectors to a minimum (data transfer to a Forward Buffer and run control messaging) on GRETA-facing interfaces. This security design is also well-aligned with the goal of reducing the complexity of re-deploying GRETA when moving between sites. The only systems that need to be reconfigured when GRETA is moved are the few systems that have management interfaces, and the Science DMZ[8] interface of the Data Transfer Node.

\section{The GRETA Data Pipeline}

\subsection{Functional components}

The GRETA data pipeline is composed of a series of components, each fulfilling its role in the transport and processing of data. The streaming design of the data pipeline means that data processing is done online, before the data are written to storage. The interface between the detector electronics (specifically the FPGA-based Signal Filter Boards) and the data pipeline is standard Ethernet[9]. In fact, the entire computing environment uses standard systems and networking technologies which are commonplace in today's world, including software capabilities such as Docker containers to simplify deployment and open source software to reduce development effort. This allows GRETA to take advantage of billions of dollars of investment in performance, reliability, and economies of scale which have followed the dramatic expansion of the Internet.

Figure 2 provides a graphical representation of the data flow through the pipeline. The data is sent from the Signal Filter Boards to the Forward Buffers. The Forward Buffers, hosted on standard servers with high-speed interfaces, receive the data and place it in queues (the internals of the Forward Buffer are further described in Section 3.2), which allows the Event Processing Cluster to self-schedule its tasks by fetching data asynchronously from the Forward Buffers. This is a key architectural point: if the Forward Buffers were not present then the Signal Filter Boards, which are built around FPGAs, would have to distribute their data to the individual cluster nodes. FPGAs are not well-suited to buffering data, or managing many network connections to a set of cluster nodes. However, the FPGAs in the Signal Filter Boards can easily send stateless messages to high-performance Forward Buffer nodes, the Forward Buffer nodes can easily manage large memory buffers to ensure that no data is lost, and the individual Event Processing Cluster nodes can easily fetch data to process when they have available cycles. Both the Signal Filter Boards and the Event Processing Cluster can thus operate asynchronously. By incorporating high-performance networking into the Signal Filter Boards, the complexity of the FPGA programming can be dramatically reduced - the Forward Buffers provide the necessary adaptation between the Signal Filter Boards and the cluster. The Forward Buffer component also allows data from auxiliary detectors to be incorporated into the data flow - the auxiliary detector sends its data to a Forward Buffer, and the Forward Buffer serves it to the Event Processing Cluster just as it would serve the GRETA HPGe data.

The cluster nodes process the data to solve for the position and energy of interaction points within a given detector crystal (the cluster node software is described in Section 3.3). The 


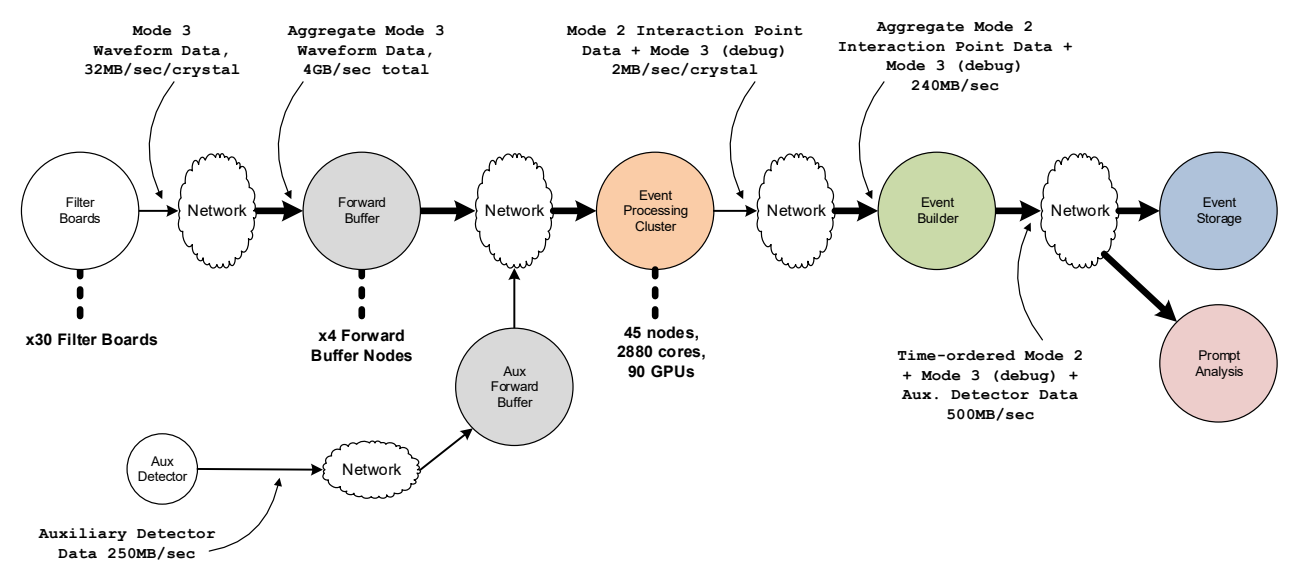

Figure 2. The GRETA data pipeline: the data enters from the detector electronics (Filter Boards or Auxiliary Detectors), and passes through each component on its way to prompt analysis and non-volatile storage. Data rates given are for maximum (aggregate) design rate of $480 \mathrm{k}$ events/sec.

architecture makes the implicit assumption that this processing operation is only dependent on the contents of the message itself and independent of future or past messages. This is the case with GRETA as each event from each crystal is independent in terms of locating interaction points. As can be seen in figure 2, the data processing step in GRETA reduces the data volume by an order of magnitude. This data reduction, combined with the ability to analyze processed data in near real time, points to the efficacy of a streaming system design in this application.

Once the interaction points for a given event in a particular crystal have been determined, the results are sent to the Event Builder. In order to perform Compton tracking across the detector array, hits from individual HPGe detectors must be correlated into global events using a hardware-based timestamp. The Event Builder is the central aggregation point in the system - until the Event Builder, all the data pipeline functions are composed of a set of parallel components which operate independently. The Event Builder orders the processed data by timestamp (this is further described in Section 3.4), and sends the data to the Event Storage component for archiving. The Event Builder also provides a subset of the data as complete events to a Prompt Analysis component which provides near-real-time feedback to the experimenter.

Communication between the pipeline components (except for the stateless communication from the Filter Board to Forward Buffer which uses UDP[10]) uses the GRETA Application Protocol (GAP), a lightweight messaging protocol built on the high-performance open source messaging protocol Nanomsg[11] which uses TCP[12] for reliability. Nanomsg was chosen over other similar technologies (ZeroMQ[13] and gRPC[14]) because in our evaluation we found that Nanomsg was more predictable in its behavior than ZeroMQ and gRPC. In addition, Nanomsg is very efficient, employing zero-copy for increased performance. GAP makes use of the REPREQ communication pattern for connection establishment, and both REPREQ and RAW for message delivery. GAP is based on the notion of Routing Headers that are embedded into each message at the beginning of the data payload. Routing Headers contain a type and a sub-type identifying detectors and types of messages, the high precision timestamp of the message, a monotonically increasing sequence number, size of data and a checksum. Routing Headers are propagated through the computing pipeline. 


\subsection{The Forward Buffer}

The role of the Forward Buffer is to interface between the FPGA-based Filter Boards and the Event Processing Cluster. The Forward Buffer allows both the Filter Boards and the Event Processing Cluster to self-schedule, because the Forward Buffer holds the data in memory until a cluster node fetches it for processing. The Forward Buffer is also able to monitor for packet loss and other anomalies (such as queue fill above threshold) and report that information to the control system. Because all messages are independent at this stage of the pipeline, the Forward Buffer is easily horizontally scalable. The GRETA design calls for four Forward Buffer nodes for 120 detectors, but this could easily be scaled higher if the experiment environment required it (see Section 4 for performance data).

\subsubsection{FPGA interface}

In order to simplify the networking stack of the Filter Boards (which use FPGAs), the Filter Boards only send UDP packets to the Forward Buffer. Because UDP is uni-directional and stateless, the Filter Boards do not need to maintain state, greatly simplifying the Filter Board implementation.

The sequence number in the routing header is used to detect message loss, and the Forward Buffer communicates message loss to the control system. The Forward Buffer also fulfils a flow control function in the presence of anomalies (e.g. a detector running faster than expected, or signal decomposition running slower than expected) - if its queues fill beyond a high water mark, it can notify the control system, allowing the detector to be synchronously throttled in a way that lessens the negative impact on a running experiment (in contrast to missing parts of events). This flow control mechanism allows an accurate accounting of the dead time introduced by any rate anomalies, in the event that such anomalies occur.

\subsubsection{Event Processing interface}

The Forward Buffer maintains a queue of messages for each detector. The Event Processing cluster queries the Forward Buffers for messages on a per-detector basis. This communication uses GAP, as described above. Multiple Event Processing cluster nodes may query the Forward Buffers for data from a single detector - this is important, because under some experiment configurations the per-detector message rate across the GRETA array can vary considerably, and more cluster resources must be devoted to the higher-rate detectors (see Section 3.3).

\subsubsection{Scalability}

The Forward Buffer pre-allocates a dedicated memory queue and UDP receiver to each detector, which means each detector is given a unique UDP port for sending data to the Forward Buffer. This allows the Forward Buffer to parallelize packet processing, and thus avoid packet loss in the general case. Similarly, the Forward Buffer allocates a GAP instance for each detector to manage communication with the Event Processing Cluster. This gives the Forward Buffer a dedicated queue per detector, with a UDP reciever on one end and a GAP instance distributing the data on the other end. This configuration can be updated at the start of each run.

The Forward Buffer thus implements a dedicated streaming path for each detector, from the Signal Filter Board through to a set of workers in the Event Processing Cluster. Each detector's streaming path can be assigned to a different Forward Buffer node during the setup 
of the experiment to balance the load of detectors across the Forward Buffer nodes. The maximum detector rate in the GRETA environment requires four Forward Buffer nodes, but if higher loads were anticipated it would be straightforward to simply add additional Forward Buffer nodes to increase the capability of the system. The ability to assign the streaming path for each detector to the appropriate Forward Buffer node provides the GRETA system with easy horizontal scalability. The Forward Buffer performance is further described in Section 4.

\subsection{Event Processing}

One of the key requirements in a GRETA experiment is the ability to determine the location of gamma-ray interaction points within the detector volume to within a few millimeters in near real time. This is the job of the event-processing cluster. One of the main problems that the event-processing cluster solves is the load imbalance (up to 7:1) between the message streams from the different detector crystals in the detector array. To address this, a compute node in the event-processing cluster is partitioned into job slots, where each job slot can be configured independently for each run. This allows the experimenter to allocate more job slots to fast-running detector crystals with a high load. Because each message can be processed independently and each job slot process messages asynchronously, the event-processing cluster can scale horizontally based on the requirements of the experiment.

In this context, a compute node refers to a Docker container with dedicated compute resources. In the GRETA environment this is typically a single multi-core CPU, a GPU accelerator, as well as memory and $\mathrm{I} / \mathrm{O}$, but the design allows for other configurations as well. The software running on the node can be divided into two categories: node management and workers.

\subsubsection{Node Management}

The node management software is independent of the specific event processing task and implements the job slot framework. The interface between the node management and the workers is kept as simple as possible. At the top level, the node management software consists of a node client that manages job slots and provides configuration and monitoring. The configuration service configures individual job slots based on a top-level configuration for the node. The control service receives control messages, while the monitoring service presents local statistics collected from the workers to the outside for harvesting.

\subsubsection{Worker Tasks}

Workers run in job slots and perform the actual event processing in the GRETA pipeline. We allocate one worker per job slot, and the worker runs as a single thread. In the GRETA environment we have two distinct workers. The signal decomposition worker connects to the Forward Buffer and receives messages that contain time-dependent signals from a single detector crystal. Multiple signal decomposition worker threads asynchronously share a single GPU by scheduling tasks on separate execution streams. While each signal decomposition worker only works on a single crystal, many workers can work on messages from the same crystal. Since each message can be processed independently at this stage, the resources required to perform signal decomposition scales linearly with the expected message rate. Once a block of messages has been received by the worker, it solves an optimization problem per message, using a model of the detector to calculate the expected signal from a set of interaction points. This is done in two different ways. First, we perform a grid search, where the 
expected signal is calculated for each combination of interaction points. This is a task that can be easily vectorized, and we use a GPU for this stage. Second, once we have a set of close matches from the grid search, we perform a conjugate gradient descent to refine the result and arrive at a set of interaction points within the required precision. Once completed, the result is enqueued on a shared queue that sorts messages based on the timestamp of the message.

The second type of worker shares a set of queues with all the signal decomposition workers and is responsible for sending large batches of sorted messages to the Event Builder. This is done to reduce the work done by the Event Builder, since this component is more difficult to scale horizontally than the Event Processing component.

\subsection{The Event Builder}

The event builder is the point in the system where the dedicated per-detector streams converge. It collects the individual processed interaction points (referred to in this section as "local events" because they are events within a single detector crystal) from the Event Processing Cluster, orders them by timestamp, and sends the ordered data stream to the Storage Service which stores the data in files.

Because the Event Builder is reading from multiple sources (a set of cluster nodes) and ordering a large volume of streamed messages in real-time $(480,000 \mathrm{msgs} / \mathrm{sec}$ at full design capacity), the sorting implementation must be highly parallel and also highly optimized. The algorithm is a hybrid of merge sort and bucket sort, and is optimized to reduce lock contention between parallel threads. In the GRETA environment, the Event Builder maintains 50,000 buckets, each served with its own Go routine (the Event Builder is written in Go[15]). In addition there are other routines necessary for handling local events, running GAP, and so on. This high degree of parallelism allows the Event Builder to make effective use of modern multi-core processors.

In addition, the Event Builder detects and builds global events composed of groups of messages from the event processing cluster. These groups are based on a common hardware timestamp generated by the electronics subsystem and contained in the Routing Header. These global events are used to reconstruct the full tracks of the gamma rays through the GRETA detector, and a subset of the events are sent to the Prompt Analysis service which provides real-time display to the experimenter. The real-time display allows the experiment team to monitor the health of the experiment and make optimizations.

\section{Performance Results}

We have characterized the performance of prototype implementations of the three performance-critical components of the system: the Forward Buffer, the Event Processing Cluster, and the Event Builder. The components were tested using realistic, synthetic data at maximum design rates. The results are described in the following subsections.

\subsection{Forward Buffer}

The performance of a Forward Buffer node is driven by the size and rate of messages coming from the detectors and constrained by the number and speed of processor cores of the node. The machine running the Forward Buffer in the performance measurement had two $3.2 \mathrm{GHz}$ Intel Xeon Gold 6146 processors (12 cores/24 hyperthreads each) and 196GB of RAM.

Table 1 shows that the larger messages are, the lower the maximum message rate is. This table also indicates that when messages are larger than the maximum size of an Ethernet 


\begin{tabular}{|l|c|c|}
\hline Message size & $\begin{array}{c}\text { Maximum message rate } \\
\text { (Single stream) }\end{array}$ & $\begin{array}{c}\text { Maximum streams } \\
\text { at 20k messages / sec }\end{array}$ \\
\hline 100 bytes & $\geq 400 \mathrm{k}^{*}$ & 50 \\
\hline 500 bytes & $\geq 400 \mathrm{k}^{*}$ & 50 \\
\hline $1 \mathrm{kB}$ & $270 \mathrm{k}$ & 50 \\
\hline $5 \mathrm{kB}$ & $210 \mathrm{k}$ & 50 \\
\hline $8 \mathrm{kB}$ & $172 \mathrm{k}$ & 50 \\
\hline $8.8 \mathrm{kB}$ & $170 \mathrm{k}$ & 50 \\
\hline $9.2 \mathrm{kB}$ & $110 \mathrm{k}$ & 20 \\
\hline $20 \mathrm{kB}$ & $80 \mathrm{k}$ & 18 \\
\hline $40 \mathrm{kB}$ & $50 \mathrm{k}$ & 16 \\
\hline $64 \mathrm{kB}$ & $28 \mathrm{k}$ & $*$ \\
\hline
\end{tabular}

Table 1. Forward buffer performance as a function of message size. Values marked by * are limited by traffic simulator performance.

jumbo frame, which gives a 9000-byte IP packet in the GRETA network, performance drops significantly due the cost of fragmenting messages into multiple Ethernet packets.

This table also shows the maximum number of detectors that can be handled by a single Forward Buffer node at message rate of $20 \mathrm{~K}$ messages per second. Note that the maximum number is 50 streams for many message sizes - this is because the dominant cost is message rate rather than data rate for message sizes that fit in a single Ethernet frame. Again, performance drops when the message size is larger than what can fit a single Ethernet frame. This is most obvious when comparing the performance of $8.8 \mathrm{kB}$ messages vs. $9.2 \mathrm{kB}$ messages - a difference of 400 bytes in message size reduces the performance by more than a factor of 2 .

The scalability and performance of the forward buffer vindicates the design choice of allocating a dedicated streaming path for each detector. Also, the highly parallel nature of the system allows for efficient use of modern multi-core processors. The Forward Buffer design is clearly able to meet the GRETA system requirement - an aggregate of 480k messages/sec across 120 detector streams with a maximum per-stream message rate of $15 \mathrm{k}$ messages $/ \mathrm{sec}$ with four Forward Buffer nodes.

\subsection{Event processing}

Figure 3 shows the performance of the GRETA event processing algorithm when the number of job slots on a single compute node is increased beyond the number of physical cores available on the node. This particular node has a single AMD EPYC 7601 CPU with 32 physical cores, but with the Simultaneous multithreading (SMT) feature, it presents 64 logical cores or threads. As the number of job slots is increased up to full occupancy, the performance (measured in $\mu$ s per event per physical core) decreases slightly. However, running one job slot per logical core shows the best performance at approximately $5 \mathrm{~ms}$ per event per core. With this performance and near linear scalability on high core count processors we can meet the event processing requirement for GRETA (480k messages processed / sec) with a relatively small number (45) of nodes.

\subsection{Event Builder}

The Event Builder's performance was measured using a simulation of 120 detectors. The aggregate message rate is $500 \mathrm{~K}$ messages per second and the maximum per-detector message 


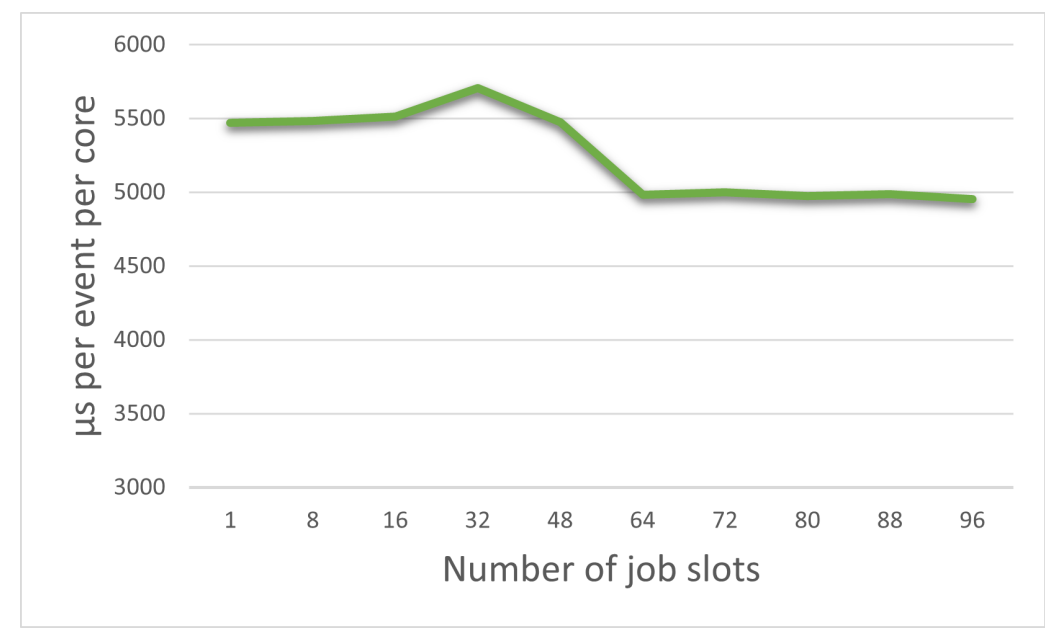

Figure 3. Node-level performance of the GRETA event-processing algorithm using a 32-core AMD EPYC 7601 CPU. Performance is measured in $\mu$ s per event per physical core.

rate is $20 \mathrm{~K}$ messages per second. This measurement was conducted using a maximum message size of 9000 bytes, ensuring messages always fit within a single Ethernet jumbo frame. This is appropriate for the GRETA environment, and shows that the Event Builder design meets the requirements of the GRETA system. The Event Builder node processors are two $3.2 \mathrm{GHz}$ Intel Xeon Gold 6146 processors (12 cores/24 hyperthreads each). When running with this workload, all cores are at $80 \%$ utilization.

\section{Conclusions}

The GRETA data pipeline design allows for the online streaming analysis of event-based data in a simple and scalable way. By introducing network-based Forward Buffers to mediate communication between the FPGA-based electronics system and the computing cluster, which performs real-time data analysis and data reduction, it is possible to apply standard network protocols and libraries to the task. The buffers allow both the electronics and computing cluster to operate asynchronously, independently writing to and reading from their respective queues, as well as providing a mechanism for global flow control. As these forward buffers handle the data streams prior to data reduction they must be capable of supporting high sustained bandwidth. This has been demonstrated in prototyping as described in Section 4.

Two other important aspects of the pipeline are data analysis and event building. To meet the event processing requirements for GRETA, multiple cores and GPU accelerator resources must be applied to each detector. This required the construction of a node-level framework which provides multiple job slots that can be allocated in accordance with the loads presented by a given experimental configuration. Finally, to enable gamma-ray tracking across all detectors in the array, events must be time correlated by a global Event Builder. This is an aggregation point in the system and as such is performance sensitive. In an early prototype of this component we have shown a sustained throughput of 500k messages / sec which meets the performance requirements of the GRETA array.

While the GRETA streaming system is purpose-built for the analysis problem faced in gamma-ray spectroscopy, we believe the architecture and pipeline components have wider 
applicability. The simple, asynchronous interface to electronics and the use of multiple forward buffers allow the system to scale to very high data rates. For systems that do not require global event building, the output of the cluster could be written directly to a parallel file system, further enhancing scalability. In addition, the forward buffers and the computing cluster it feeds do not need to be co-located, which opens up for the possibility that the system can be used in a distributed or Superfacility[16] context, where the cluster is replaced by HPC resources at a remote facility. Finally, the network-centric design will allow the system to take advantage of the rapid evolution of hardware and software stacks in this space.

\section{References}

[1] Gamma ray energy tracking array (greta), http://greta.lbl.gov/ (2021), accessed: 202102-11

[2] Facility for rare isotope beams (frib), https://frib.msu.edu/ (2021), accessed: 2021-0211

[3] Argonne tandem linac accelerator system (atlas), https://www.phy.anl.gov/atlas/ facility/index.html (2021), accessed: 2021-02-11

[4] I. Lee, Nuclear Instruments and Methods in Physics Research Section A: Accelerators, Spectrometers, Detectors and Associated Equipment 422, 195 (1999)

[5] M. Deleplanque, I. Lee, K. Vetter, G. Schmid, F. Stephens, R. Clark, R. Diamond, P. Fallon, A. Macchiavelli, Nuclear Instruments and Methods in Physics Research Section A: Accelerators, Spectrometers, Detectors and Associated Equipment 430, 292 (1999)

[6] P. Fallon, A. Gade, I.Y. Lee, Annual Review of Nuclear and Particle Science 66, 321 (2016), https://doi .org/10.1146/annurev-nucl-102115-044834

[7] Epics - experimental physics and industrial control system, https://epics-controls.org/ (2021), accessed: 2021-02-12

[8] E. Dart, L. Rotman, B. Tierney, M. Hester, J. Zurawski, The Science DMZ: A network design pattern for data-intensive science, in SC'13: Proceedings of the International Conference on High Performance Computing, Networking, Storage and Analysis (2013), pp. 1-10

[9] Ieee 802.3-2018 - ieee standard for ethernet, https://standards.ieee.org/standard/802_ 3-2018.html (2021), accessed: 2021-02-12

[10] J. Postel, RFC 768, RFC Editor (1980), http://www.rfc-editor.org/rfc/ rfc768.txt

[11] Nanomsg, https://nanomsg.org/index.html (2021), accessed: 2021-02-23

[12] J. Postel, RFC 793, RFC Editor (1981), http://www.rfc-editor.org/rfc/ rfc793.txt

[13] Zeromq, https://zeromq.org/ (2021), accessed: 2021-05-07

[14] grpc, https://grpc.io/ (2021), accessed: 2021-05-07

[15] Golang, https://golang.org/ (2021), accessed: 2021-05-18

[16] B. Enders, D. Bard, C. Snavely, L. Gerhardt, J. Lee, B. Totzke, K. Antypas, S. Byna, R. Cheema, S. Cholia et al., Cross-facility science with the Superfacility Project at LBNL, in 2020 IEEE/ACM 2nd Annual Workshop on Extreme-scale Experiment-in-theLoop Computing (XLOOP) (2020), pp. 1-7 